\title{
GINGA OBSERVATIONS OF ACTIVE GALACTIC NUCLEI
}

\author{
H. Inoue \\ Institute of Space and Astronautical Science \\ 9-1-1, Yoshinodai, Sagamihara \\ Kanagawa 229, Japan
}

\begin{abstract}
Ginga observed 116 AGNs during its operational life from 1987 to 1991: Among them, there were 55 Seyfert galaxies, 42 quasars, 9 BL Lac objects and 10 other AGNs, although the classification is not strict. From these AGN observations, a number of fruitful results were obtained. In this review, I briefly summarize the Ginga observations of AGNs and show several similarities between the Seyfert-type AGNs and the galactic black hole candidates. I also discuss two inverse correlations between the break energy of the power law spectrum and the flux observed from the black hole candidate GS2023+338 and between the equivalent width of the iron fluorescent line and the continuum flux observed from NGC4151.
\end{abstract}

\section{Summary of the Ginga observations}

The results of the Ginga observations are summarized as follows (for the general review of the X-ray observations of AGN, see Mushotzky, Done and Pines 1993):

\subsection{SPECTRAL SLOPE}

Statistically significant X-ray spectra have been obtained from many of the above AGNs by Ginga. The systematic study of the spectral properties of Seyfert galaxies (Awaki 1991; Nandra 1991) show that the 2-30 keV spectrum of the most of Seyfert galaxies can be approximated by a simple power law form and the specral energy indices distrubute in a fairly narrow range around 0.7 (see e.g. Mushotzky, Done and Pines 1993). The mean spectral index of QSOs observed with Ginga was separately investigated and found to be still higher than that of the cosmic X-ray background (Williams et al. 1992).

The determination of the mean spectral index of AGNs fainter than the Ginga detection limit can also be addressed by analyzing the source confusion noise in the LAC background (Hayashida 1990). From one pointing position to another, the integrated flux of these faint sources fluctuates in the LAC field of view. An analysis which obtained the mean spectral index of the source confusion noise has been performed and the result shows that the spectral energy-index is about 0.8 . This spectral slope is still significantly steeper than that of the cosmic X-ray background. The mean flux of these objects is $5-10 \times 10^{-13}$ ergs $\mathrm{cm}^{-2} \mathrm{~s}^{-1}$.

\subsection{REFLECTION COMPONENT IN SPECTRA}

Ginga observations of AGN established that the presence of an iron emission line in the spectrum is common to almost all Seyfert galaxies. The line center energies obtained from the Ginga observations of Seyfert galaxies are almost all consistent with the $\mathrm{K}_{\alpha}$-line energy of neutral iron, after we take account of the cosmological

T. J.-L. Courvoisier and A. Blecha: Multi-Wavelength Continuum Emission of AGN, 73-82.

(C) 1994 IAU. Printed in the Netherlands. 

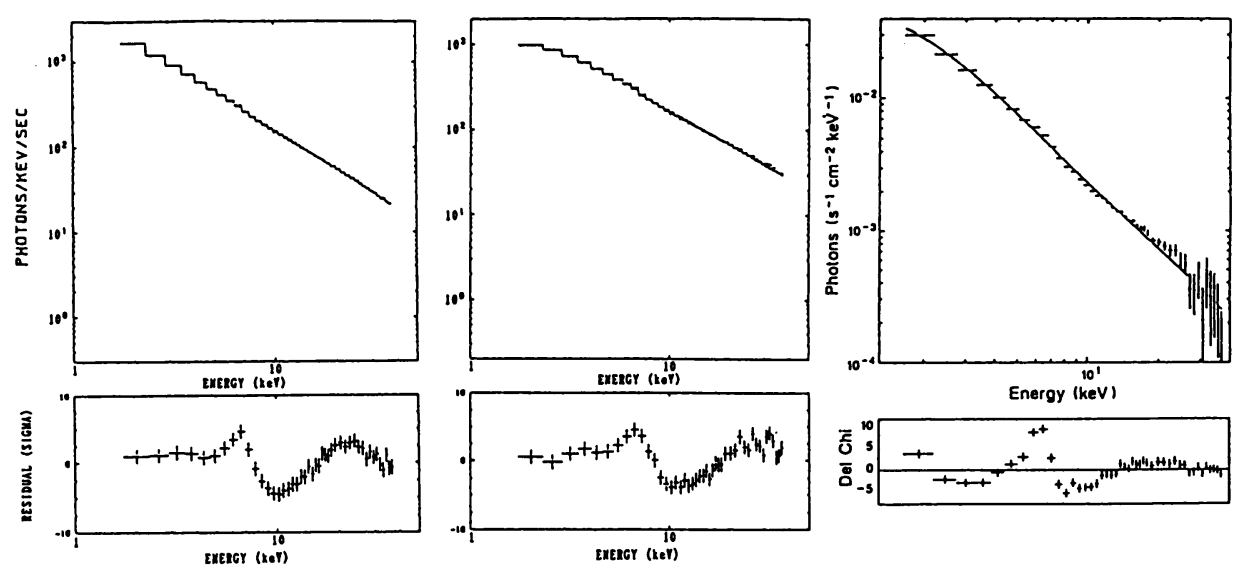

Fig. 1. Simple power law fits to the spectra in the hard state (upper panel) and residual plots of the data minus model results (lower panel) for Cyg X-1 (left), GS2023+338 (center), and an average of twelve active galactic nuclei (right, Pounds et al. 1990) observed with Ginga.

redshift of the line energy. If the iron-line-emitting matter subtends the central $\mathrm{X}$-ray source with a large solid angle, $\mathrm{X}$-rays reflected from the iron-line emitting, cold matter should be observed in the spectrum simultaneously with the fluorescent iron line. In fact, Ginga observations of AGNs revealed that the spectrum generally shows a shallow and broad absorption like feature at energies from 7 to $15 \mathrm{keV}$ together with the emission line feature at 6-7 keV. Furthermore, the power law continuum tends to flatten at higher energies (Pounds et al. 1989). These features are very similar to those observed from black hole candidates as shown in Fig.1.

These spectral features can be reproduced very well by a superposition of a component reflected by cool Thomson-thick matter from a single power law. The emission line feature at 6-7 keV, the shallow and broad absorption feature in the 7-15 keV range and the excess above $15 \mathrm{keV}$ are all consistent with the presence of a reflection component in these spectra.

\subsection{SEYFERT I AND SEYFERT II GALAXIES}

Seyfert II galaxies which Ginga observed generally showed evidence of a large obscuration of the central engine by cool matter. In particular, Ginga detected an intense iron line with an equivalent width as large as $1.3 \mathrm{keV}$ from a Seyfert II galaxy, NGC1068 (Koyama et al. 1989). An equivalent width as large as $1-2 \mathrm{keV}$ can be expected only when the direct X-rays from the central source are blocked by dense matter while the $\mathrm{X}$-rays reprocessed by cold matter around the central engine are still observed. 


\subsection{IRON EMISSION LINES FROM QUASARS}

Ginga has discovered the presence of iron emission lines in the spectra from two bright quasars, 3C273 (Turner et al. 1990) and 1E1821+643 (Kii et al. 1991).

The equivalent width of the line from a radio quiet quasar $1 \mathrm{E} 1821+643$ is 180 $\mathrm{eV}$ a value similar to that from Seyfert I galaxies. This distant $(z \simeq 0.3)$ radioquiet quasar probably has a geometry similar to those of nearby Seyfert galaxies as discussed above. On the other hand, significant evidence of an iron line from a radio loud quasar $3 \mathrm{C} 273$ was found only when $3 \mathrm{C} 273$ was very dim, and the equivalent width was only $50 \mathrm{eV}$. Since $3 \mathrm{C} 273$ is known to exhibit superluminal motion, the line of sight to the central engine in $3 \mathrm{C} 273$ can be considered to be very close to the direction of the mass ejection, according to the canonical interpretation of superluminal motion. The weak iron line from 3C273 would imply that the X-ray beaming in the direction of the mass ejection is relatively strong in radio-loud/OVV quasars exhibiting superluminal motion. In fact, none of the OVV quasars observed from Ginga shows a significant iron line.

\subsection{BL LAC OBJECTS}

A simultaneous ROSAT-Ginga observation has clearly shown that the X-ray spectrum of a BL Lac object, Mrk 421, has a concave shape: providing support for synchrotron interpretation (Makino et al. 1991). A systematic relation is found between the intensity and the spectral hardness during the flux variation in the sense that harder X-rays always precede softer X-rays: another support for direct synchrotron emission for steep spectrum (index > 1.5) BL Lac objects (Sembay et al. 1993; Tashiro 1993). The time lag has been measured for some sources. Lack of iron emission line has been confirmed for all of the measured objects with high statistics (Kii et al. 1992), which is consistent with synchrotron model with the continuum emission likely to be boosted by a relativistic motion.

\section{Similarities between AGNs and Galactic Black Hole Candidates}

There are several similarities between AGNs and galactic black hole candidates (BHC: for the review, see e.g. Tanaka 1992; Inoue 1991) as shown below.

\subsection{POWER LAW SPECTRUM}

The power law slope, 0.5 - 1, of the Seyfert galaxies is very similar to that of the BHC in the hard state (see e.g. Tanaka 1992). This suggests that there is a common physical mechanism to produce a power-law spectrum with an index of 0.5 - 1 in the central power houses, independently of the wide range of the mass of the central power house from 10 solar mass probably up to $10^{8}$ solar mass.

The spectral slope also seems to be independent of the wide range of the accretion rate onto the central power house. In the case of the Galactic transient source, GS2023+338, the slope stayed almost constant despite the large flux change of over three orders of magnitude. Similarly, the spectral slope of the OVV quasar 3C279 
did not change greatly even when the source flared up by a factor of $>10$ (Makino et al. 1989).

\subsection{REFLECTION COMPONENT IN SPECTRA}

Ginga observations of BHC have revealed that the reflection component in the spectrum, as observed from AGN, is also generally observed from galactic black hole candidates (see Fig.1). Figure 1 shows residual plots of the observed spectrum minus a single power-law model for Cyg X-1 and GS2023+338; the presence of these features is evident.

The similarity of AGN to BHC in the presence of the reflected component in the spectrum strongly suggests that an accretion disk exists in the central engine of AGN. It is generally believed that accreted matter will form an accretion disk in binary X-ray sources and the outerpart of the disk will be responsible for the $\mathrm{X}$-ray reflection.

\subsection{SPECTRAL BREAK AROUND $100 \mathrm{KEV}$}

Recent hard X-ray observations by Mir-Kvant, GRANAT-SIGMA and GRO-OSSE revealed that the power law spectrum of both Seyfert galaxies and galactic black hole candidates doesn't extend to the energies higher than $100 \mathrm{keV}$ but breaks around there (see e.g. Maisack et al. 1993; Salotti et al. 1992).

\subsection{TIME VARIABILITY}

Similarities between AGN and BHC exist also in their time variabilities.

The power spectra of X-rays from BHC such as Cyg X-1 and GS2023+338 have the logarithmic slope of power density against frequency $\sim 0$ in the frequency range below about $10^{-1.5} \mathrm{~Hz}, \sim-1$ in the range between $10^{-1.5} \mathrm{~Hz}$ and $10^{0.5} \mathrm{~Hz}$, and $\sim-2$ in the range above about $10^{0.5} \mathrm{~Hz}$ (see e.g. Inoue 1988). On the other hand, those of AGNs such as NGC5506 and NGC4051 obtained by Pounds and McHardy (1988) have remarkably similar properties except that the "knee" frequencies are $10^{4}-10^{5}$ times lower.

If the similarities in the power spectra among these sources come from a common energy generation mechanism in the central power houses, the differences of the "knee" frequencies may be interpreted as differences of the typical dimension of the central power house, which will be proportional to the central mass. The masses of AGNs estimated by scaling the "knee" frequencies from the mass of Cyg $\mathrm{X}-1$ (Paczynski 1974) are roughly proportional to the X-ray luminosities (Pounds and McHardy 1988 and see also Barr and Mushotzky 1986; Wandel and Mushotzky 1986).

These similarities over the wide range of the mass of the central object suggest the following scalings:

The structure of the accretion flow should be similar to all AGNs and galactic black hole candidates if the distance-scale is normalized by the Schwartzschild 


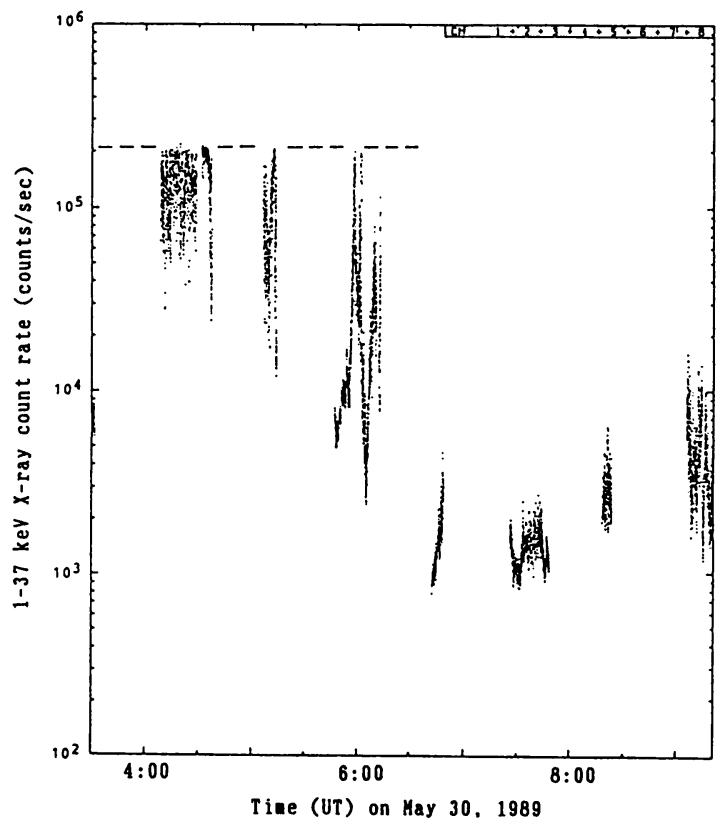

Fig. 2. X-ray light curve of GS2023+338 on May 30 in 1989 observed with Ginga. A dashed line indicates a possible luminosity saturation.

radius which is proportional to the central mass.

When the quantity, $L / L_{\mathrm{E}}$, (the luminosity, $L$, normalized by the Eddington limit, $L_{\mathrm{E}}$ ) is the same, the physical situation around the black hole would be the same over a wide range of the central mass.

If the particle energy is proportional to the depth of the potential energy, it will be independent of the central mass at the position close to the Schwartzschild radius. Then, the fact that the emergent photon spectrum has the same functional form of the energy independently of the central mass seems to suggest the photon emission mechanism to be thermal and optically thin.

The following discussions are consistent with these suggestions.

\section{Inverse Correlation between Break Energy and Luminosity}

Ginga discovered the bright transient source GS2023+338 (Kitamoto et al. 1989) and performed several observations during its decay. The follow-up optical observation revealed this source to be probably a black hole (Casares, Charles and Naylor 1992). As seen in the X-ray light curve of GS2023+338 on May 30, 1989, in Fig.2, the flux apparently saturated at the peak of the outburst. For the dis- 


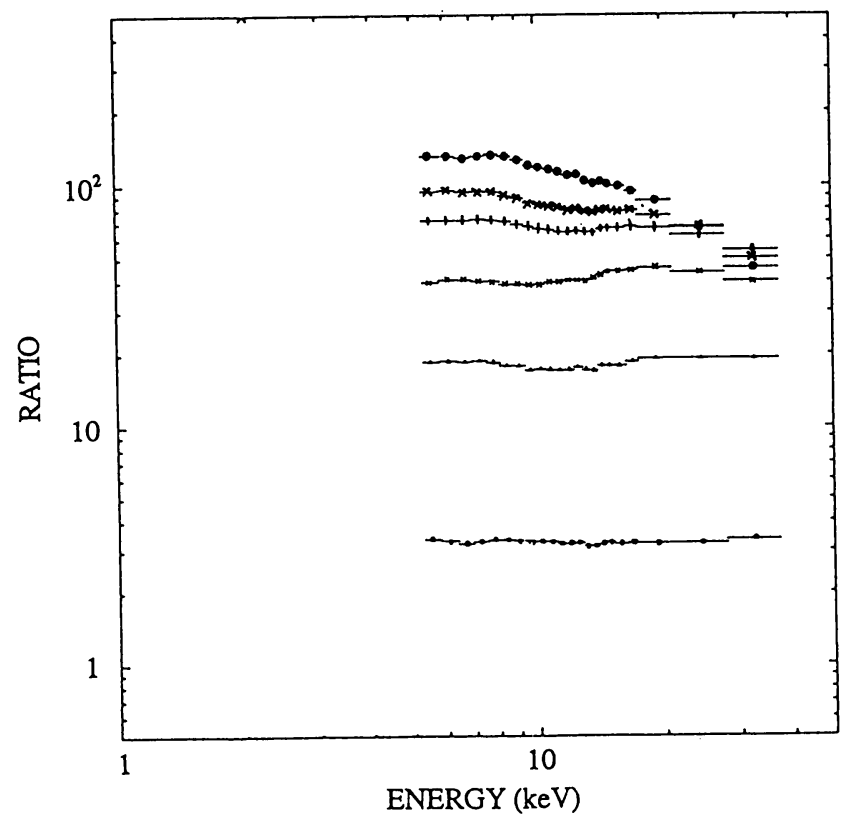

Fig. 3. Ratios of the spectra of GS2023+338 obtained at different flux levels to a typical power law spectrum at a certain flux level.

tance of 1-3 kpc evaluated from the optical observations (Casares et al. 1991), the saturation level is consistent with the Eddington limit of a several-solar-mass star. GS2023+338 showed a single power law spectrum with a reflection component throughout the decay by over three orders of magnitude, although the source sometimes suffered from heavy absorption and revealed a complicated spectrum during the first ten days after the discovery.

Figure 3 shows the ratios of the spectra obtained at different flux levels to a typical power law spectrum at a certain flux level. The spectrum at the highest flux level was obtained when the luminosity was very close to the saturation level probably corresponding to the Eddington limit. It is clearly seen in Fig. 3 that the spectra when the flux is very bright reveals a break around $10 \mathrm{keV}$ and the break energy seems to shift to the higher energy as the flux decreases. The shift of the break energy becomes clear by introducing the observations of this source by the Mir-Kvant Observatory (Sunyaev et al. 1991). The Kvant observations were done when the flux was about a 50th to a 200th of the highest flux of the Ginga observations. At those flux levels, the break was seen around $100 \mathrm{keV}$. The break energies at the various flux levels observed by Ginga and Mir-Kvant are roughly estimated and are plotted in Fig.4. The inverse correlation between the break 


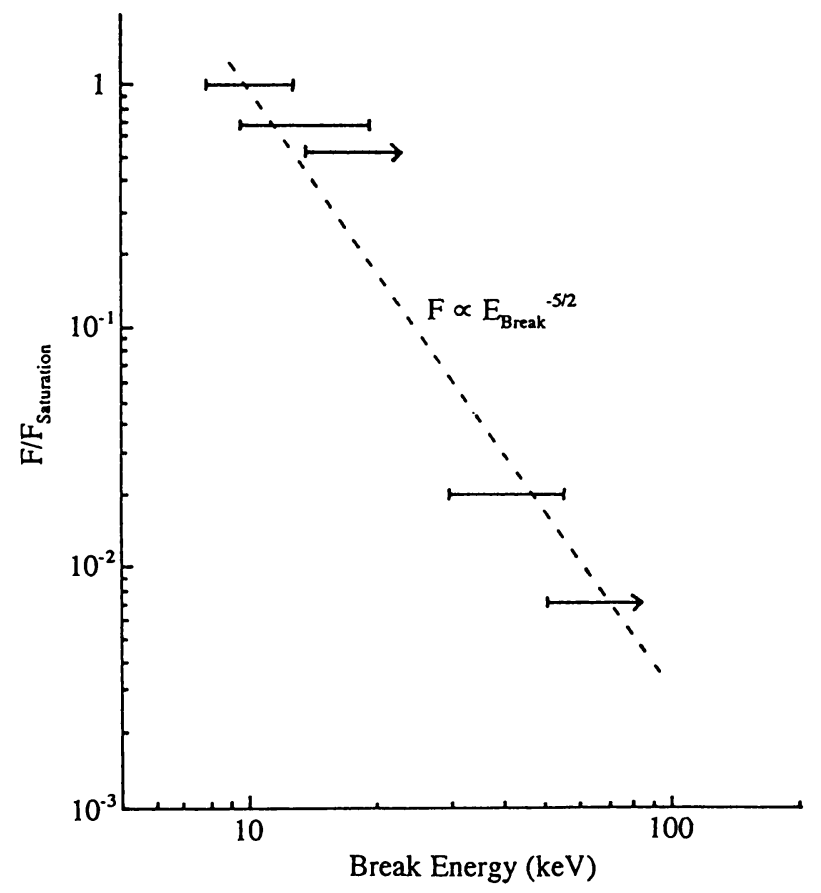

Fig. 4. Break energy of a power law spectrum observed at the various flux levels normalized by the saturation level of GS2023+338 by Ginga and Mir-Kvant (Sunyaev et al. 1991).

energy and the flux is obvious. This inverse correlation can be interpreted by a simple consideration as follows.

Let's consider a situation that an optically thin, geometrically thick accretion disk surrounds a central black hole. Here, the geometrically thick accretion disk is assumed to be an spherical hot plasma with the radius of $R$. The plasma will be heated up by the gravitational energy release of the accretion flow through some possible mechanism such as viscous heating or solar flare-like activity. Then the proton temperature, $T_{\mathrm{p}}$, will approximately be given by $k T_{\mathrm{p}} / m_{\mathrm{p}} \simeq G M / R$, where $k, m_{\mathrm{p}}, G$ and $M$ are the Boltzmann constant, the proton mass, the gravitational constant and the mass of the central black hole respectively. Since the energy loss rate of protons as discussed below is estimated to be sufficiently small compared to the energy input rate through the accretion, the proton temperature is assumed to be constant in the followings.

The protons will give their energy to electrons through two body collisions and the energy loss rate per unit volume is given as $(3 / 2) n k T_{\mathrm{p}} / t_{\mathrm{pe}}$ if $T_{\mathrm{p}} \gg T_{\mathrm{e}}$, where $n$ and $T_{\mathrm{e}}$ are the number density and the electron temperature. $t_{\mathrm{pe}}$ is the relaxation time for protons and electrons to be in the thermal equilibrium and is 
proportional to $\left(k T_{\mathrm{p}} / m_{\mathrm{p}}+k T_{\mathrm{e}} / m_{\mathrm{e}}\right)^{3 / 2} / n$ (see e.g. Spitzer 1962), where $m_{\mathrm{e}}$ is the electron mass. This energy flow heats up electrons while the electrons loose their energy through Compton scatterings with ambient photons. The cooling rate per a unit volume is approximately given as $\left(4 k T_{\mathrm{e}} /\left(m_{\mathrm{e}} c^{2}\right)\right) f n \sigma$, where $c$ is the velocity of light, $f$ is the photon flux and $\sigma$ is the electron scattering cross section. $f$ is roughly related to the luminosity, $L$, as $f \simeq L \tau /\left(\pi R^{2}\right)$ (when $\tau>1$ ) or $L /\left(\pi \mathrm{R}^{2}\right.$ ) (when $\tau<1$ ), where $\tau \simeq n \sigma R$.

This simple consideration for the energy flow from protons to photons can explain two important observational facts.

1) Since the luminosity should be equal to the total energy loss rate of the electrons in the volume of $4 \pi R^{3} / 3$, we obtain $y \simeq 1$ by equating $\left(4 k T_{\mathrm{e}} /\left(m_{\mathrm{e}} c^{2}\right)\right)$ fn $\sigma\left(4 \pi R^{3} / 3\right)$ with $L$. Here, $y$ is the so called Comptonization parameter defined as $y=\left(4 k T_{\mathrm{e}} /\left(m_{\mathrm{e}} c^{2}\right)\right) \tau$ (when $\left.\tau<1\right)$ or $\left(4 k T_{\mathrm{e}} /\left(m_{\mathrm{e}} c^{2}\right)\right) \tau^{2}$ (when $\left.\tau>1\right)$. If $y$ is close to unity, the emergent photon spectrum is expected to be a power law with the energy index of about 1 (see e.g. Shapiro, Lightman and Eardley 1976; Sunyaev and Titarchuk 1980).

2) In the steady state, the energy flow rate from protons to electrons should balance with the flow rate from electrons to photons. By equating $(3 / 2) n k T_{\mathrm{p}} / t_{\mathrm{pe}}$ with $\left(4 k T_{\mathrm{e}} /\left(m_{\mathrm{e}} c^{2}\right)\right) f n \sigma$, we obtain a simple relation $T_{\mathrm{e}} \simeq\left(L / L_{\mathrm{E}}\right)^{-2 / 5}$ when $\tau>1$ and $k T_{\mathrm{e}} / m_{\mathrm{e}}>k T_{\mathrm{p}} / m_{\mathrm{p}}$. This relation can roughly explain the inverse correlation between the break energy and the luminosity observed from GS2023+338. The weak dependency of the break energy on the normalized luminosity could also explain the recent observational evidence that the hard X-ray spectra of Seyfert galaxies as well as galactic black hole candidates seem to generally break from the simple power law spectrum around $100 \mathrm{keV}$, if the luminosities of these black hole candidates are well below the Eddington limit.

\section{Inverse Correlation between Iron Line Equivalent Width and Flux}

Iwasawa and Taniguchi (1993) have recently pointed out the inverse correlation between the equivalent width of the iron line and the luminosity for the Seyfert galaxies based on the Ginga observations. Figure 5 shows the relation of the equivalent width with the continuum flux for NGC4151 (Yaqoob and Warwick 1991) and the inverse correlation is obvious.

We shall assume that a geometrically thin, optically thick accretion disk surrounds the central hot region discussed above.

The equivalent width of the fluorescent iron line will be proportional to the solid angle of the line reproccessing region as viewed from the central $\mathrm{X}$-ray emitter. If the central X-ray emitter has the height of $R$ and the reprocessor has a negligibly small thickness, the solid angle of the disk further than a radius, $r$, is approximately given as $2 \pi R / r$. The innermost radius, $r$, of the line reproccessing disk should be determined by the so called ionization parameter $\xi$. When $\xi$ is sufficiently larger than $10^{3}$ erg $\mathrm{cm}$, the iron will be fully ionized and emits no line (Kallman and 


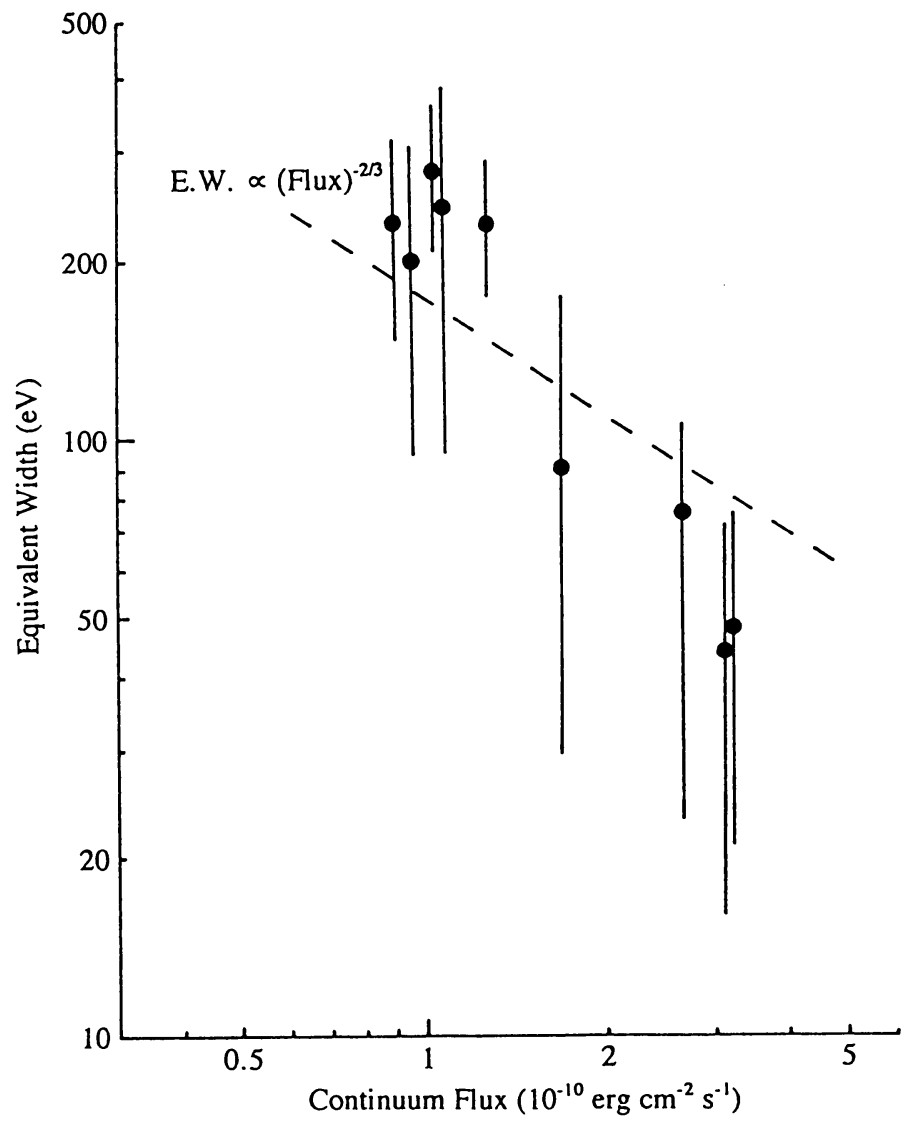

Fig. 5. Relation of the equivalent width of the fluorescent iron line with the continuum flux for NGC4151 (Yaqoob and Warwick 1991).

McCray 1982). Since X-rays graze the cool disk, the $\xi$ should be redefined as $\left(L / n r^{2}\right)(R / r)$. Furthermore, the region responsible for the line reprocessing will be only a surface layer with the column density, $n h$, of about $10^{24} \mathrm{H}_{\text {atoms }} \mathrm{cm}^{-2}$, where $h$ is the thickness of the surface layer. Hence, $\xi$ can be further rewritten as $(L / n h r)(h / r)(R / r)$. Since $h / r$ can be approximated by $\left(k T / m_{\mathrm{p}}\right)^{1 / 2} r^{1 / 2}$, we finally obtain $\xi \propto T^{1 / 2}\left(R_{\mathrm{S}} / R\right)^{1 / 2}(n h)^{-1}\left(L / L_{\mathrm{E}}\right)(r / R)^{-3 / 2}\left(R_{\mathrm{S}}\right.$ is the Schwarzshild radius). If the innermost radius of the line reprocessing region is given at the place where $\xi$ is about $10^{3-4} \mathrm{erg} \mathrm{cm}, T$ is about $10^{6-7} \mathrm{~K}$ and $R$ is constant, $(r / R)$ is in proportion to $\left(L / L_{\mathrm{E}}\right)^{2 / 3}$. Since the equivalent width $\propto(R / r)$ as discussed above, the equivalent width of the reprocessed iron line is expected to be proportional to $\left(L / L_{\mathrm{E}}\right)^{-2 / 3}$. This expectation is roughly consistent with the inverse relation 
between the equivalent width and the flux obtained from NGC4151 as seen in Fig.5. The non-dimensional form can also explain the common presence of the reflected component in the spectrum over the wide range of the mass of the central black hole. (For the recent more quantitative treatment for the surface layer of the line reprocessing geometrically thin disk, see Ross and Fabian 1993; Matt, Fabian and Ross 1993).

\section{References}

Awaki, H. 1991, Ph.D.Thesis, Nagoya Univ.

Barr, P. and Mushotzky, R.F.: 1986, Nature, 320, 421.

Casares, J., Charles, P.A. and Naylor, T. 1992, Nature, 355, 614.

Casares, J. et al. 1991, M.N.R.A.S., 250, 712.

Hayashida, K. 1990, Ph.D.Thesis, Univ. of Tokyo.

Inoue, H. 1988, in "Two Topics in X-ray Astronomy" , (ESA, SP-296), 783.

Inoue, H. 1991, in "Frontiers of X-Ray Astronomy" ed. Y.Tanaka and K.Koyama, (UAP, Tokyo), 291.

Iwasawa and Taniguchi 1993, Ap. J. (Letters), 413, L15.

Kallman and McCray 1982, Ap. J. Suppl., 50, 263.

Kii, T. et al. 1991, Ap. J., 367, 455.

Kii, T. et al. 1991, in "Frontiers of X-Ray Astronomy" ed. Y.Tanaka and K.Koyama, (UAP, Tokyo), 577.

Kitamoto, S. et al. 1989, Nature, 342, 518.

Koyama, K. et al. 1989, P.A.S.J., 41, 731.

Maisack, M. et al. 1993, Ap. J. (Letters), 407, L61.

Makino, F. et al. 1989, Ap. J. (Letters), 347, L9.

Makino, F. et al. 1991, in "Frontiers of X-Ray Astronomy" ed. Y.Tanaka and K.Koyama, (UAP, Tokyo), 543.

Matt, G., Fabian, A.C. and Ross, R.R. 1993, M.N.R.A.S., 262, 179.

Mushotzky, R.F., Done, C and Pounds, K.A. 1993, Ann. Rev. A. Ap., 31, 717.

Nandra, K. 1991, Ph.D.Thesis, Univ. of Leicester.

Paczynski, B. 1974, A. Ap., 34, 161.

Pounds, K.A. and McHardy, I.M. 1988, in "Physics of Neutron Stars and Black Holes" ed. Y. Tanaka, (UAP, Tokyo), p.285.

Pounds, K.A. et al. 1990, Nature, 344, 132.

Ross, R.R. and Fabian, A.C. 1993, M.N.R.A.S., 261, 74.

Salotti, L. et al. 1992, A. Ap., 253, 145.

Sembay, S. et al. 1993, Ap. J., 404, 112.

Shapiro, S.L., Lightman, A.P. and Eardley, D.M. 1976, Ap. J., 204, 187.

Spitzer, L.Jr. 1962, Physics of Fully Ionized Gases (Wiley, New York).

Sunyaev, R.A. and Titarchuk, L.G. 1980, A. Ap., 86, 121.

Sunyaev, R.A. et al. 1991, Sov. A. Lett., 17(2), 123.

Tanaka, Y. 1992, in "Proceedings of the Ginga Memorial Symposium" ed. F.Makino and F.Nagase, (ISAS, Sagamihara), 19.

Tashiro, M. 1993, Ph.D.Thesis, Univ. of Tokyo.

Turner, M.J.L. et al. 1990, M.N.R.A.S., 244, 310.

Wandel, A. and Mushotzky, R.F. 1986, Ap. J. (Letters), 306, L61.

Williams, O.R. et al. 1992, Ap. J., 389, 157.

Yaqoob, T. and Warwick, R.S. 1991, M.N.R.A.S., 248, 773. 